\title{
Malgorata Maria Pastian: Tanzen. Über die Bewegung die Seele heilen
}

HKIT, 2019, Schneverdingen, 176 Seiten, 15,00€

פTheir

anzen - Über die Bewegung die Seele heilen “ ist eine Einführung in die Biodynamische Tanz- und Körpertherapie Heilende Kräfte im Tanz (HKIT). Die Autorin Malgorzata Maria Pastian, seit 2009 in Hamburg in eigener Praxis als Tanztherapeutin tätig, erläutert detailliert Methoden und wissenschaftliche Grundlagen, ergänzt um Erfahrungsberichte ihrer KlientInnen.

In acht Abschnitten werden die spezifischen Herangehensweisen der Tanztherapie HKIT erklärt. Jedes Kapitel bildet eine in sich abgeschlossene Einheit, dadurch ist es möglich, innerhalb des Buches zu springen. Die Autorin lässt sich von einer Hauptfrage leiten: „Was ist alles in unserem Leben passiert, was uns davon abgebracht hat [...], unser schöpferisch-kreatives Sein an der Quelle der Lebendigkeit zu verbringen?“" (S. 28)

Nach der Einführung in die theoretischen Grundlagen widmet sich Malgorzata Pastian dem Heilungstanzritual Aatini. Weitere Themen sind Trauma und Trauma-Transformation, die Arbeit mit dem Inneren Kind, der positive Umgang mit Gefühlen und die Stärkung der Selbstheilungskräfte. Ein Interview mit Gabriele Fischer, der Begründerin der Methode HKIT, ergänzt und vertieft die Gedanken der Autorin. Das Schlusskapitel beinhaltet die tänzerische Arbeit im Wasser und ihr therapeutisches Potential.

Malgorzata Pastian gelingt es, auf einem hohen sprachlichen und wissenschaftlichen Niveau laienverständlich die Komplexität ihrer Arbeit und der Methode HKIT zu vermitteln, immer unter Angabe der von ihr verwendeten Literatur und Quellen. Auch für „Profis“ ist die Lektüre empfehlenswert.

\section{Sabine Rippe}

DOI 10.2378/ktb2021.art14d 